Hongbo Wang

Wuliu Tian

Jinfen Zhang

Yongjiang $\mathrm{Li}$

http://dx.doi.org/10.21278/brod71202

\title{
A HYBRID SELF-ORGANIZING SCHEDULING METHOD FOR SHIPS IN RESTRICTED TWO-WAY WATERWAYS
}

UDC 629.5.05:629.5.07:629.5.077.1

Original scientific paper

\begin{abstract}
Summary
Traffic conflicts between ships are one of the most important reasons causing delays in restricted waterways. Aiming to improve the traffic efficiency, a hybrid self-organizing scheduling (HSOS) method for restricted two-way waterways is proposed. Ship transportation system is treated as a distributive and self-organized system under uncertainties. Each ship makes the decision on when to enter the waterway and how to keep the safe distance between them, while the VTS center could manage the direction of traffic flow according to the navigation situations. In order to reduce the traffic conflict between the opposite directions, small ships are given higher priority than the large ships in the same direction. When the large ships are accumulating, they are given higher priority than small ships in the same direction. The large ships are delayed while small ships decrease the waiting time. The trade-off between small and large ships can enhance efficiency by accumulating the large ships. Comparing the results from HSOS with First Come First Served (FCFS), it can effectively reduce the average delays brought by large ships, especially at high arrival rates.
\end{abstract}

Key words: $\quad$ restricted waterways; traffic efficiency; ship scheduling; self-organizing

\section{Introduction}

As one of the most important transportation modes, shipping is increasing with the requirement of world and domestic trade in terms of volume and size [1,2]. This brings some challenges to traffic efficiency in some busy waterways, especially the restricted waterways [3]. Some ships have to wait a long time with long queues, which would result in high economic loss. Enhancing the waterway infrastructure can be regarded as one of the most effective ways to alleviate such problem. However, huge infrastructure investment on waterways is not practical in many real cases. Another way is enhancing the efficiency to promote the utility of waterway resource. Ship scheduling is a common way and is an indispensable component in maritime transportation management. However, as an intuitive scheduling methods widely applied to queue problems, First Come First Serve (FCFS) is not the most efficient one because it prioritizes equality rather than efficiency. 


\subsection{Optimizing scheduling method}

Scheduling is the process of creating a queue of the arriving ships to determine how to order them and how to allocate resources considering the variety of possible objectives. The procedure of scheduling is usually deemed as an optimization procedure. From this perspective, the majority of scheduling methods rely on a "classical" or Cartesian mode of thinking, which is expressed most explicitly in the classical or Newtonian mechanics [4]. Related studies concentrates on ship scheduling in one-way waterways. It is assumed that the arrival time and speed of every ship are available. Berthing and traveling directions are the main constraints while the objective of scheduling strategy is minimizing the average or overall waiting time. For example, Zhang et al. [5] established a mathematical model by coordinating channels and berths. The results show that the algorithm can ensure safety and improve efficiency simultaneously. Furthermore, Zhang et al. [6] added the minimum total waiting time as the objective, and then the ship scheduling become a multi-objective optimization problem. The Pareto Optimum solutions were selected as the ship scheduling schemes. However, the presumption that the speed of behind ship could not exceed that of ahead is not practicable because the behind ship can keep the safe distance by reducing its speed in real cases [7]. Liang et al. [8] proposed a method to sort vessel sequence for the controlled waterways in the upper Yangtze River, where Fuzzy Analytical Hierarchy Process (FAHP) was adopted to establish a hierarchical mode and calculate the weights of influencing factors. The expert system was employed for data processing and reasoning. The results showed that the waiting time of vessels can decrease to a large degree. Nevertheless, the performance of the vessel scheduling is not reliable owing to the subjective judgments from different experts.

With the increasing of ships navigating in waterways, one-way waterways cannot adjust to the maritime transportation and are being replaced by two-way waterways. In practice, the two-way traffic can be regarded as the combination of two one-way traffic flow and the conflicts in two-way waterways can be addressed in accordance with the methods been applied in one-way waterways. However, the dimensions of some ships are very large, so that the two-way transportation is not allowed in many cases. As a result, the traffic mode usually switches between one-way and two-way transportation. In dealing with this issue, a sequential ship traffic scheduling approach [9] was presented. The proposed method can restrain the waiting time at an acceptable level, especially when the proportion of large ships is remarkable. It should be noted that an assumption is made that all the ships are navigating under identical and stable speed in the model. However, the speed of ships usually varies a lot within certain ranges in many real cases [10]. Also, every ship was assumed to arrive at the entrance at the corresponding time reported at least two hours in advance in the approach. It should be noticed that many ship incidents such as collision avoidance operations occurs and cannot be predicted precisely. Hence, it is not practicable that all the ships are assumed to arrive at the expected time.

\subsection{Self-organizing scheduling method}

From a mathematical point of view, traffic scheduling is a non-deterministic polynomial-time hard (NP-hard) problem and it is not possible to find an analytical solution. Moreover, many existing methods do not consider the real time traffic status. The optimization methods are over constrained to "unusual" situations. For example, some ships change speed or alter course for some reasons [11]. Besides, the rate of arriving ships also fluctuates during different periods. Self-organization [12] is another method for solving such problems. It is a process that the global order arises from local adjustment among the ships. The ships that have effect on each other make coordination in an initially unordered system. The organization is performed in a distributed mode over all the components of the system. 
Ships usually make decisions individually on the basis of local information and the conflicts are alleviated to some extent. In this sense, waterway transportation should also be treated as a distributive and self-organizing system [13]. Based on the idea of self-organization, the traffic system is complex and unpredictable in essence. But the unexpected events could be modelled in a self-organizing mode.

Many self-organization approaches have been investigated in biology from molecular to ecosystem perspective [14]. It has been demonstrated that a biologically inspired approach can solve some of the fundamental transportation problems such as in urban areas [15]. Gershenson and Rosenblueth [16] believed that traffic organization is not so much an optimization problem, but rather an adaptation problem, since traffic flows and densities vary a lot with time and space. Adaptive methods should be regulated by the traffic flow itself. The research by Tonguz and Viriyasitavat [17] reveals that the vehicles can manage traffic in urban areas with dedicated communications radios by themselves. It has shown that the design of local rules enables the vehicles to approach an intersection to resolve the ensuing conflict in a seamless and self-organizing manner. Self-organization has been observed in ship traffic systems such as cellular automata [18]. Qu and Meng [19] proposed a modified cellular automata model for ship movements in restricted waterways and considers interactions between consecutive ships by expert judgment. Discrete event models are applied to generate vessels with different types and speeds in the Singapore Strait. Ship following rules and crossing rules are used to simulate the ships' motion in order to adapt to all types of navigational scenes.

Self-organization has also emerged in multi-agent marine system [20] while considering various navigational risks [21-23]. Vaněk et al. [24] provided a data-driven simulation model of the maritime transportation system affected by piracy. The model employed an agent-based approach. The behavior of the system is represented as a composition of a series of microlevel behaviors of individually simulated vessels. Observing that traffic efficiency is influenced by speed differences, Wang et al. [25] proposed a mathematical model of delay time in different entry sequences. On the basis of this, each ship could make a decision regarding to its own sequence to find the local optimum. In short, ships could manage conflict like vehicles in some conditions. Nevertheless, the traffic control is also vital when the contradiction among ships is irreconcilable. Therefore, a ship scheduling method fusing the self-organization with control adapts more to the real ship traffic.

Assuming that the arrival time and speed of ships are not precisely available, a novel hybrid self-organizing scheduling method is proposed in this paper to assist the ships to pass through a restricted two-way waterway to enhance traffic efficiency in an adaptive manner. The remainder of this paper is organized as follows. Delay of ships in restricted a two-way waterway is discussed in Section 2 considering the ship safety domain. The hybrid selforganizing scheduling method through traffic conversion is introduced in Section 3. The simulation experiments are performed and the results are discussed in Section 4. Finally, conclusions are made in Section 5.

\section{Problem description}

\subsection{Ship traffic system of restricted two-way waterways}

The waterway discussed in this paper is a restricted two-way waterway with two anchorages nearby the entrances, as shown in Fig. 1. It mainly includes a Vessel Traffic Service (VTS) centre, two anchorages, and the arriving ships and so on. VTS is responsible for supervising and scheduling the ship traffic from the macro perspective. The anchorages are water areas for ship to anchor, lying at both sides of the waterway. When a ship arrives at 
the approach, it will enter the anchorage, or steer into the waterway when transit is allowed and no conflict exists. All the ships obey the ship following rules until they pass through the waterway. The automatic identification system (AIS) is a trajectory tracking system installed on the ships. Information of a ship provided by the AIS equipment, such as unique identification, position, course, and speed, can be identified by others ships and VTS. By AIS data, the traffic situation and intention can be easily obtained by each ship in the system.

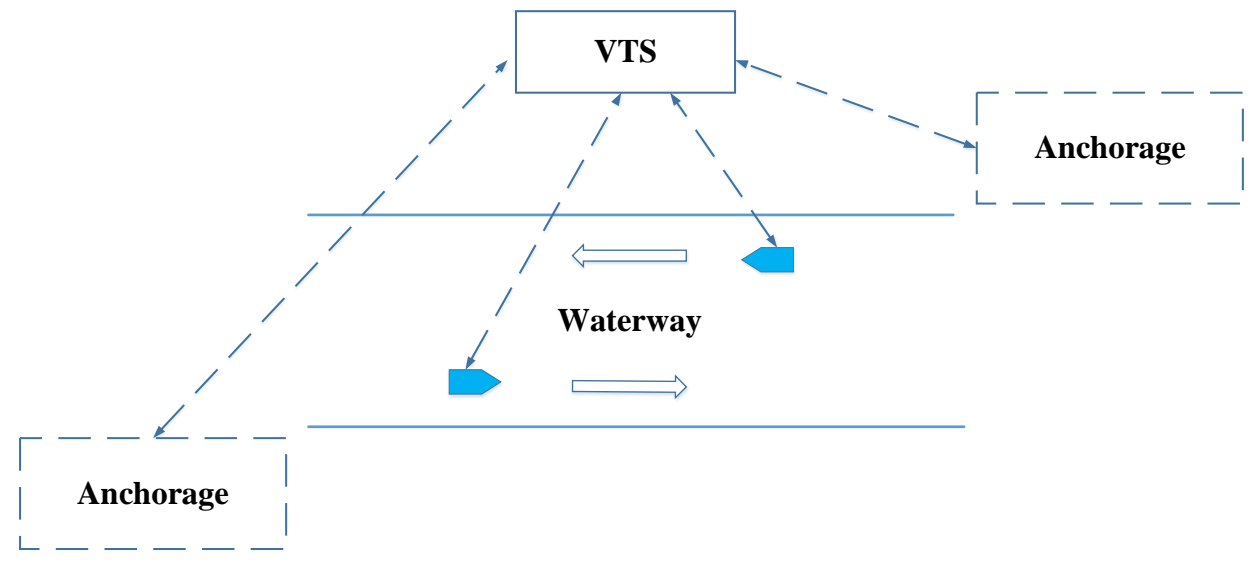

Fig. 1 Ship traffic system for restricted two-way waterway

\subsection{Conflicts between ships in opposite directions}

Overtaking is usually prohibited to all ships navigating in the restricted waterway. Moreover, ships are required to maintain safe distances between each other to avoid collisions in the waterway. Ship safety domain is a generalization of a safe distance [26] and is one of the criteria proposed for the safety of ships, particularly in restricted areas [27]. The widely adopted model is an ellipse with an offset center. It is generally accepted that the size of ship domain is closely related with ship's size. Regarding the fact that a ship conflict occurs when one ship enters another's domain [28], the influence of ship traffic in opposite directions is specially considered.

According to the size of ship domain, the ships in the waterway could be classified as small ships or large ships. The limit value for the size of a small ship depend on the width of the fairway. A demonstration is displayed in Fig. 2. A green checkmark in Fig. 2 (a) identifies the allowed situation, which is a head-on situation between two small ships. It is adequately safe for them to pass through each other with acceptable collision risk. The red error marks in Fig. 2(b) and Fig. 2(c) express that a small ship or a large ship is not allowed to enter the restricted waterway before a large ship passes through the waterway, due to the overlapping of ship domain areas. Such conflicts should be avoided in order to guarantee navigation safety. Therefore, the traffic flow in the opposite direction has to stop and wait at anchorage when a large ship is navigating in the waterway, which leads to delays, and further influences the traffic efficiency. 


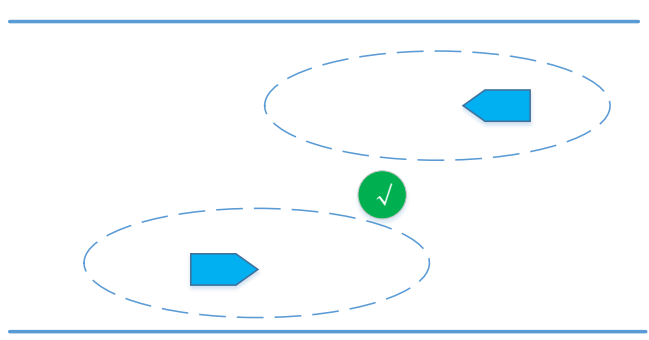

(a) allowed head-on situation between two small ships

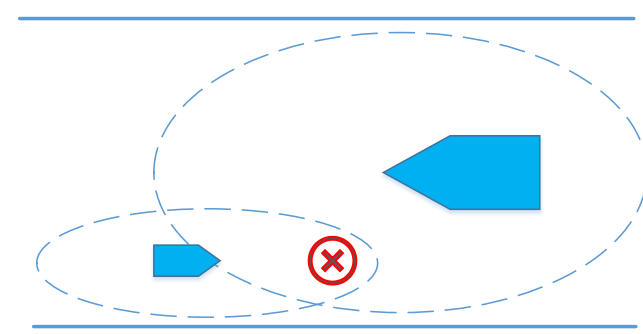

(b) forbidden head-on situation between a small ships and a large ship

(c) forbidden head-on situation between two large ships

Fig. 2 Conflict between ships in the head-on situation

\subsection{Conflicts between ships in the same direction}

Besides the above problem, each ship should also keep a safe distance away from the preceding ship in the same directions as shown in Fig. 3 (a). That is to say, the following rules should be satisfied: If a ship is faster than the preceding ship, the distance between them would gradually reduce to be smaller than the safe distance. Noting that overtaking is not allowed, the faster ship has to slow down to keep clearance with the front ship, which would also reduce traffic efficiency. The collision risk would be unacceptable if speed reduction is not performed, as presented in Fig. 3 (b).

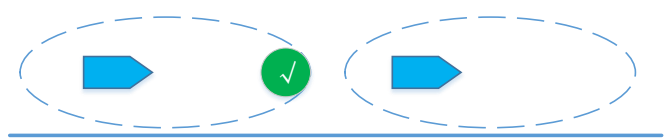

(a) following situation where the distance is larger than the safe distance

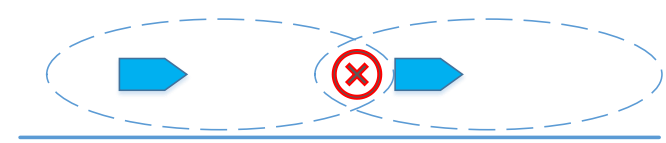

(b) following situation where a distance which is smaller than the safe distance

Fig. 3 Conflict between ships in the following scenarios

\section{Ship scheduling method}

\subsection{Assumptions}

The implementation of traffic system is based on the following assumptions:

- The ships' arrival time and speed are uncertain, which are expressed by random variables with different types of probability distributions;

- Overtaking is not allowed in the waterway; 
- All the ships are supposed to follow the traffic rules in the waterway. It should be noted that the paper only consider the ships that are regularly navigating in the main channel of waterways, not including the sailing vessels, fishing ships, or very small ships that usually have free navigation within all the waters.

- Entering the waterway is not allowed when a large ship is transiting in the opposite direction;

- VTS has the authority to control the traffic flow direction.

Ship-VTS communications have been set up and the self-adapting priority assignment is possible within ship traffic system. On the one hand, the traffic scheduling is self-adapting because the conversion of flow direction is adaptive to the current situation of traffic flow. On the other hand, the ships with different velocities are self-adapting because they slow down to keep the safe distance between each other and try to adjust to local collaboration.

In FCFS, the traffic flow is divided by the flow direction. In each direction, the ships enter the waterway on the basis of the sequence of arrival time. Before a large ship enters the waterway, the traffic flow in the opposite direction is forbidden. Once a large ship is navigating in the waterway, the ships in opposite direction have to wait in anchorage. When a small ship is navigating in the opposite direction, a large ship should also stay at anchorage for safety. As a result, long waiting time brought by large ships is one of the main causes of the delays. Special attention need to be paid to this issue.

\subsection{Hybrid self-organizing scheduling method}

Ship traffic modelling is time-consuming because there would be high probability that the large and small ships are obstructed by each other. There are many ways in which the ship traffic flow can be constrained for improving efficiency. One of them is self-organizing according to the distributed characteristics of traffic system. Rules can also be introduced to mediate among the conflicting ships by limiting or binding their behaviours. A ship scheduling mechanism based on rules will coordinate large ships to mediate between large ships and small ships to reduce the conflicts between them. Following this idea, an efficient method is proposed, named Hybrid Self-Organizing Scheduling (HSOS), which is accomplished through the control to traffic flow direction by VTS. HSOS has advantage in the trade-off between small and large ships, so that there would be coordination between the average waiting time of all the ships and the maximum waiting time. The priorities of small and large ships would change during the scheduling process, based on which these two types of ships can be scheduled efficiently. Been considered as agents, the behaviour model for small ships is presented in Tab. 1, while large ship behaviour model is described in Tab. 2.

The definitions of several logical variables are explained as follows.

$T F S$ : Is the traffic flow in the same direction allowed?

$T F O$ : Is the traffic flow in the opposite directions allowed?

LSO: Are large ships navigating in the opposite direction?

SSO: Are small ships navigating in the opposite direction?

$L S A$ : Are large ships waiting in own anchorage?

SSA: Are small ships waiting in own anchorage?

$S D$ : Is the requirement on safe distance satisfied?

OSS: Is own ship the earliest small ship in own anchorage?

$O S L$ : Is own ship the earliest large ship in own anchorage? 
Tab. 1 Small ship agent behaviour model (1 means YES and 0 means NO)

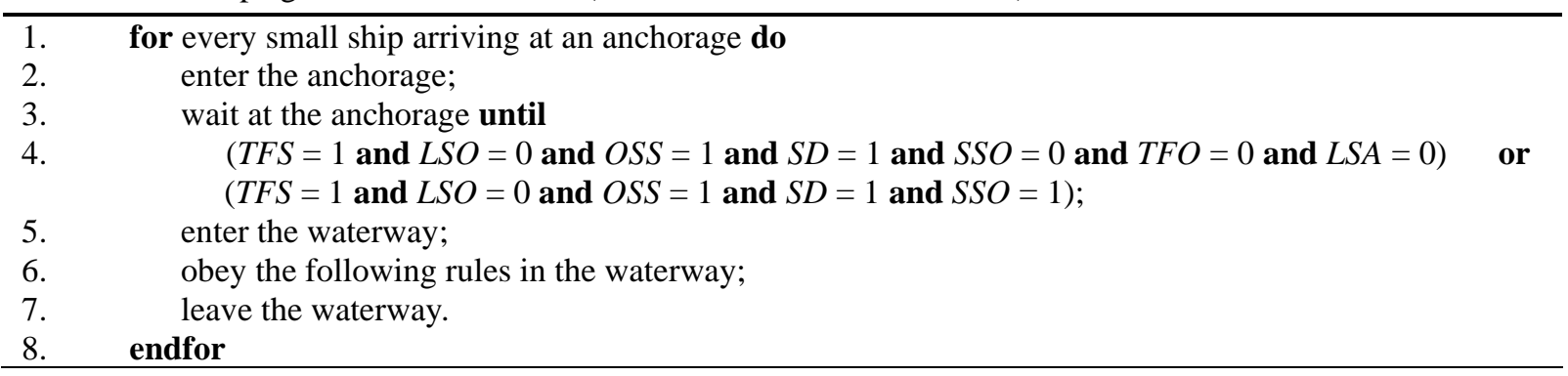

Tab. 2 Large ship agent behaviour model

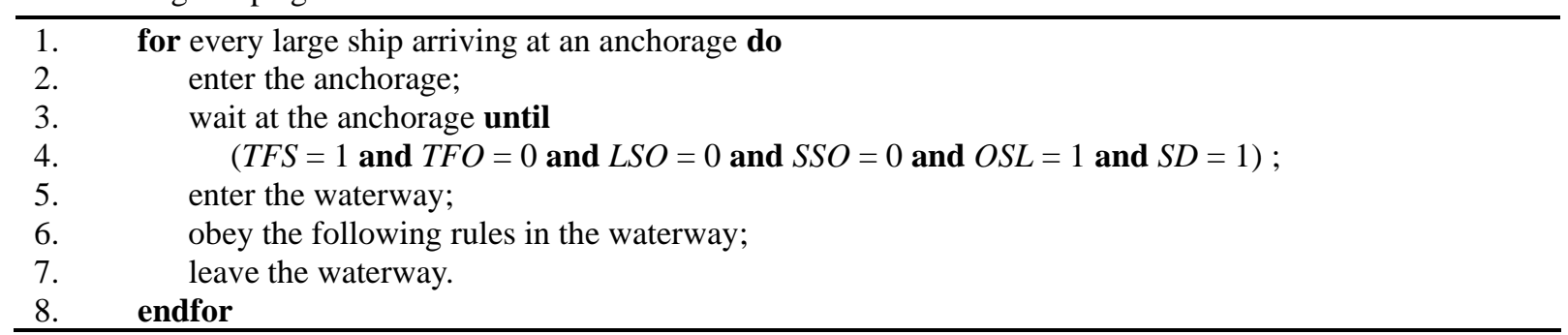

In HSOS, if the traffic flow in one direction is allowed by VTS, a small ship enters the waterway as long as there is no large ships in the opposite direction. When a large ship is at anchorage due to the impact of small ships in the opposite direction, it gives higher priority to small ships in the same direction, as demonstrated in Fig. 4 (a). Such mechanism is different with FCFS. However, if small ships have a high arrival rate, it is quite common that some small ships are usually navigating in the opposite direction while large ships have to wait at the anchorage for a very long time in HSOS. In dealing with such problem, a mechanism is designed to allow these waiting large ships to enter the waterway as follows: VTS enumerates the number of large ships waiting at an anchorage $\left(l_{i}\right)$. When $l_{i}$ reaches a threshold $T h_{l s}$, the traffic flow in the opposite direction would be closed by VTS. Once the ships in the opposite direction are clear, the large ships waiting in anchorage could enter the waterway. Furthermore, the waiting large ships are prior to the waiting small ships at the same anchorage as shown in Fig. 4 (b), so as to clear the large ships from the anchorage as early as possible. By doing this, the traffic conflict is alleviated to some extent.

Such mechanism is described in more detail in Tab. 3. It realizes self-organization in an adaptive manner as follows: if there is only a few large ships in an anchorage, allowing them entering the waterway would pose a lot of conflicts to the ships in the opposite direction. As a result, they are encouraged to wait for some time, giving opportunity to other large ships to join them. The accumulations of large ships alleviate the traffic conflicts to a large degree. Once there are enough large ships (e.g. reach the threshold $T h_{l s}$ ), the opposite traffic flow is forbidden even if the upcoming ships are arriving at the anchorage continuously. When there are no ships in the opposite direction, large ship platoon generates like the vehicles in roads [29] and enter the waterway with higher priority. 


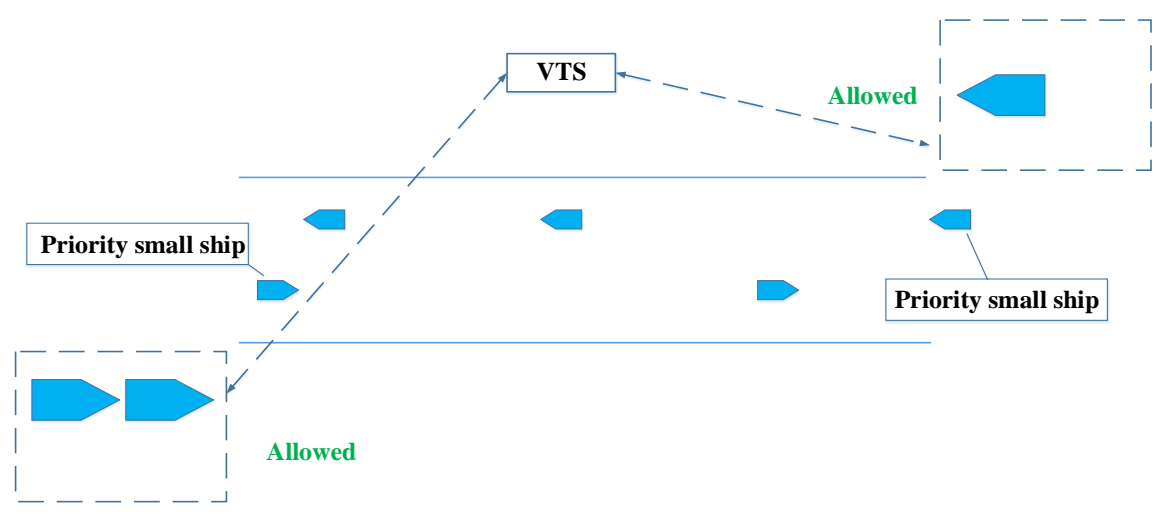

(a) The small ships are given higher priority than large ships in same direction

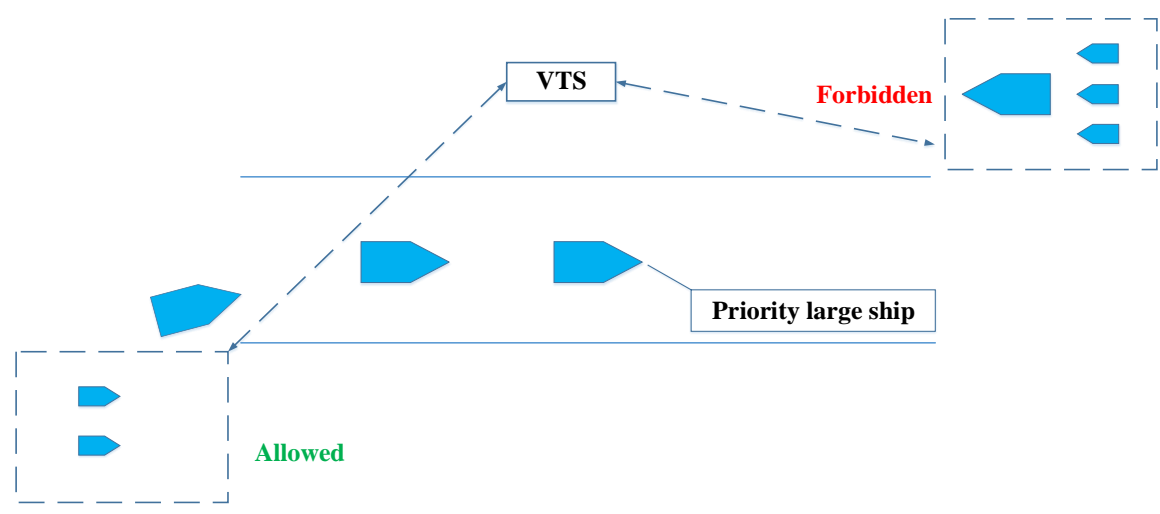

(b) When traffic flow in the opposite direction is forbidden, large ships are given higher priority than small ships in the same direction

Fig. 4 Interaction among small ships, large ships and VTS

Tab. 3 VTS agent behaviour model

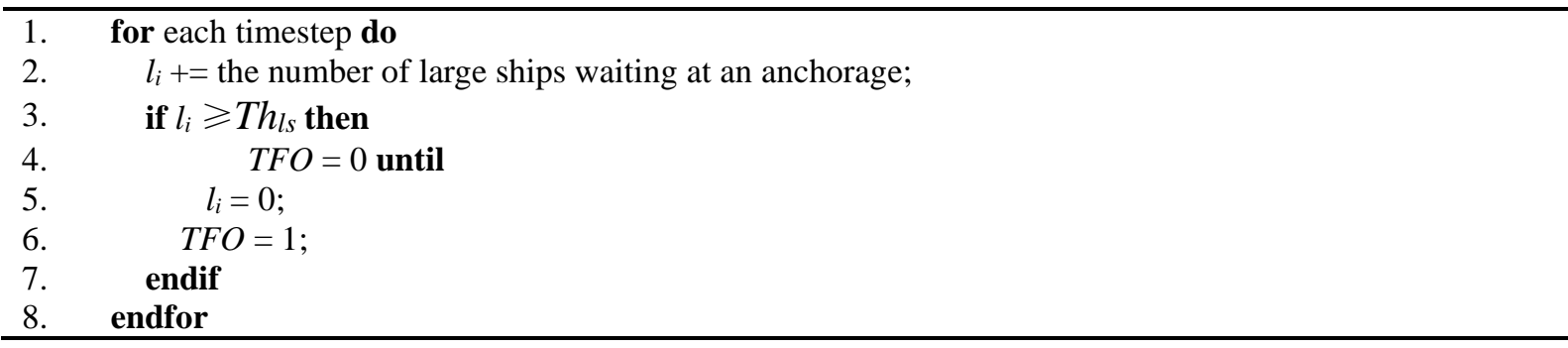

The proposed scheduling approach operates in a hybrid manner under the assumption that VTS periodically evaluates the number of waiting large ships in each anchorage and determines if a traffic flow in a direction should be forbidden. It becomes obvious that it would be useful to consider VTS as the agents as well. The objective of VTS is to keep the allowed traffic as long as possible and promote large ships waiting at anchorage to leave as early as possible. To do so, VTS should forbid traffic flow in the opposite direction when many large ships are waiting at the anchorage. VTS also monitors the large ships in the two directions, in order that the forbidden flow is resumed as soon as possible. In addition, ships operate in a distributed manner with the assistance of AIS. Every ship announces its current position and speed to other nearby ships as well as to VTS. The objective of ships is to enter the waterway as early as possible while guaranteeing safety. A ship can therefore construct a local map and determine if there is an ensuing conflict at the opposite and the same direction where it is about to enter. Since the objectives of VTS and ships are complementary, they 
interact in the manner of self-organization to achieve synergy. Finally, HSOS can be realized in an adaptive way, combining centralized control with distributed control.

\section{Experiments and analysis}

\subsection{Parameters setup}

In order to evaluate the influences of waiting and speed deceleration in a quantitative way, the parameter of delay time $T_{\text {delay }}$ is introduced to evaluate traffic efficiency, which is defined as the sum of waiting time and the increased navigation time caused by deceleration (also denoted as deceleration time) [21]. Based on the above analysis, the objective function for ship scheduling in a restricted two-way waterway is described as

$$
\text { Min } T_{\text {delay }}=\frac{1}{m} \sum_{i=1}^{m} T_{i}^{\text {delay }}=\frac{1}{m} \sum_{i=1}^{m}\left(T_{i}^{\text {wait }}+T_{i}^{\text {dec }}\right)
$$

where $m$ is the number of arriving ships, $T_{i}^{\text {delay }}$ is the delay time for ship $i, T_{i}^{\text {wait }}$ is the waiting time for ship $i$, and $T_{i}^{\text {dec }}$ is the deceleration time. According to the above model, the objective is to minimize the sum of average waiting time and deceleration time, considering that the large ships and the small ships are obstructed by each other when navigating in different directions and speeds.

Monte Carlo simulations are used to make an evaluation by the law of large numbers, which is widely applied to ship traffic studies. In order to evaluate the performance of HSOS, the software of Arena (Version 14.0), a discrete event simulation and automation software developed by Rockwell Automation, is used to construct a simulation model [30]. A 10- $n$ mile waterway is assumed with anchorages lying at the end of waterway in each side in the simulations. VTS centre is responsible for the management of traffic flow. All ships are assumed to be equipped with AIS, which could cover the whole area including the waterway and anchorages. Therefore, position, speed and direction of each ship can be obtained by other ships as well as the VTS centre. According to the number of large ships waiting at an anchorage, VTS will transmit the order of changing traffic flow direction, which would be announced to all the ships.

Some experiments are analysed on restricted two-way waterway. The results from the proposed HSOS method is compared with that from FCFS. The traffic generation pattern used in the simulations is depicted in Tab. 4. The ships are supposed to arrive at an anchorage randomly, which follows the Poisson distribution. According to the rate of arrival $R_{a r r}$, there will be on average 2 to 4 ships arriving per hour at each direction. The traffic flow follows stochastic equilibrium distribution, which means that both directions have similar traffic volume. In addition, the ratio of large ships $P_{\text {lar }}$ ranges from 0.1 to 0.3 . As far as the ship speed is concerned, normal distribution is used with the mean value of $10 \mathrm{kn}$ and the standard deviation of $1.5 \mathrm{kn}$. Considering the steerageway and regulations on maximum speed in some restricted waterways, the values of speed are confined in the range between 4 and $20 \mathrm{kn}$. The requirements on safe distances with previous ships also pose limitations to the scheduled ships, which is supposed to be $1200 \mathrm{~m}$ for simplification.

Simulations of 200-day traffic are performed in the experiments and was repeated for 10 times to provide enough samples. The warm-up period in the simulations is set to be 10 days and only the outputs in the following days are involved in data analysis. As discussed previously, the number of large ships is one of the most influencing factors in traffic conflict. It should be noticed that the number of arriving large ships is closely related with $R_{\text {arr }}$ and the proportion of large ship $P_{l a r}$. As mentioned in section 3, the threshold $T h_{l s}$ of large ships 
waiting at anchorage in each changing of direction also needs to be determined in different combination of arrival rate and large ship proportion before being compared with FCFS, so that the overall traffic efficiency can be enhanced to the maximum extent. Maximum $T h_{l s}$ is limited to 6 in order that the large ships do not need to wait too long time. In general, the main objective for the simulations is to observe the trend of ship delay times with the increment of the 3 parameters $\left(R_{a r r}, P_{l a r}\right.$ and $\left.T h_{l s}\right)$.

Tab. 4 Parameter settings

\begin{tabular}{lll}
\hline Parameter & Unit & Values \\
\hline Rate of arrival $R_{\text {arr }}$ & $h^{-1}$ & $2.0,2.5,3.0,3.5,4.0$ \\
Proportion of large ship $P_{l a r}$ & & $0.1,0.15,0.2,0.25,0.3$ \\
Traffic flow distribution & & 0.5 \\
Mean speed & $k n$ & 10 \\
Standard deviation of speed & $k n$ & 1.5 \\
Waterway length & $n$ mile & 10 \\
Safety distance & $m$ & 1200 \\
Number of replication & & 10 \\
Replication length & $d$ & 200 \\
Warm-up period & $d$ & 10 \\
Threshold & $T h_{l s}$ & $1,2,3,4,5,6$ \\
\hline
\end{tabular}

\subsection{Results and analysis}

In order to make a comprehensive analysis, a total of $5 \times 5 \times 6=150$ scenarios of the simulation runs are performed with different $R_{a r r}, P_{l a r}$ and $T h_{l s}$. Delay time of ships are collected from the simulations. It should be noted that such inputs follow some kind of probability distributions, including the arriving time intervals and speeds of ships, making the outputs fluctuant. In order to analyse the error or uncertainty in the measurement, error bars representing a $95 \%$ confidence interval are presented.

It can be seen that smaller average delay time is more favourable. The results of average delay time at the case $\left(R_{a r r}=4 h^{-1}, P_{l a r}=0.25\right)$ with different $T h_{l s}$ are presented in Fig. 5 (a). It can be seen that average delay time of large ships have a sharp increasing tendency with $T h_{l s}$, which means more average delay time of the large ships is generated with the increase of $T h_{l s}$ in HSOS method. It can be explained that a larger $T h_{l s}$ signifies that a large ship usually needs longer waiting time in order that there would be more large ships to accumulate.

Meanwhile, the average delay time of small ships have a slowly decreasing trend. It indicates that the obstructed chance of small ships decreased and traffic efficiency of small ships is promoted when large ships are grouped. In most cases, the proportion of small ship is more than that of large ships. Therefore, global traffic efficiency can be improved when $T h_{l s}$ reaches a value, even if the large ships suffer from longer delay time. For example, it is observed that the optimum appears when the number of large ships in each group is 4 , as presented in Fig. 5 (b). 


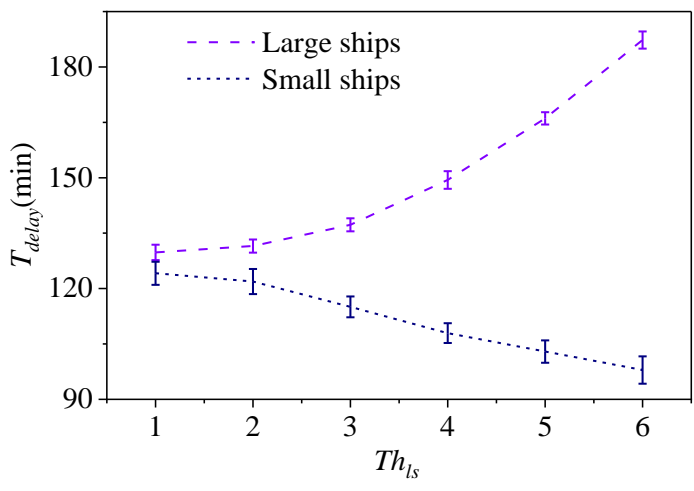

(a) $T_{\text {delay }}$ of large and small ships in $R_{\text {arr }}=4 h^{-1}$ and $P_{\text {lar }}=0.25$

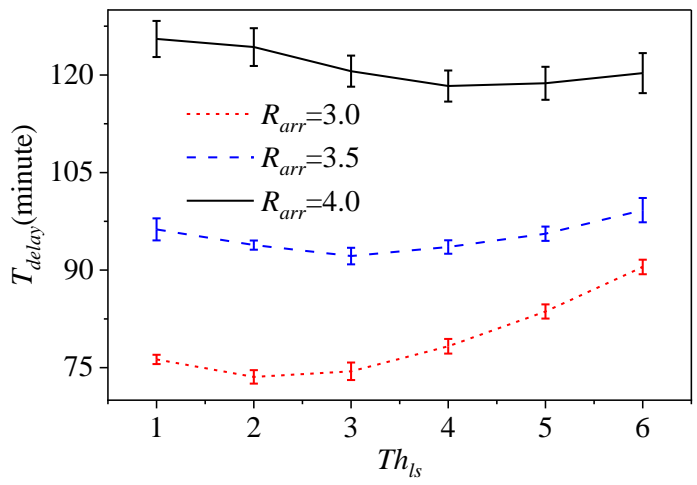

(c) $T_{\text {delay }}$ of all ships in $P_{\text {lar }}=0.25$

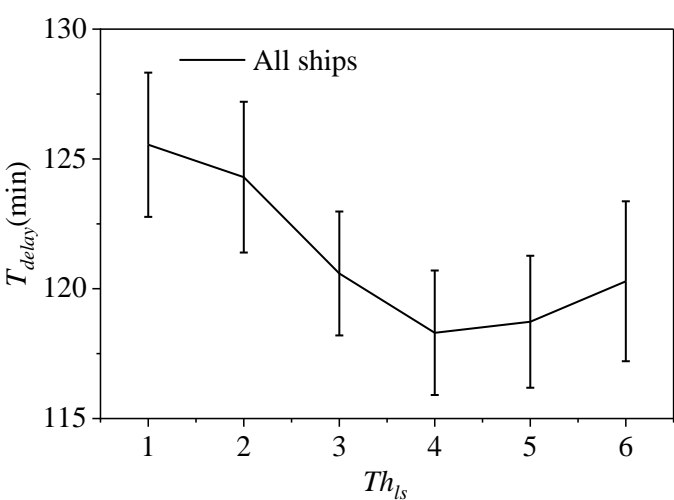

(b) $T_{\text {delay }}$ of all ships

in $R_{\text {arr }}=4 h^{-1}$ and $P_{\text {lar }}=0.25$

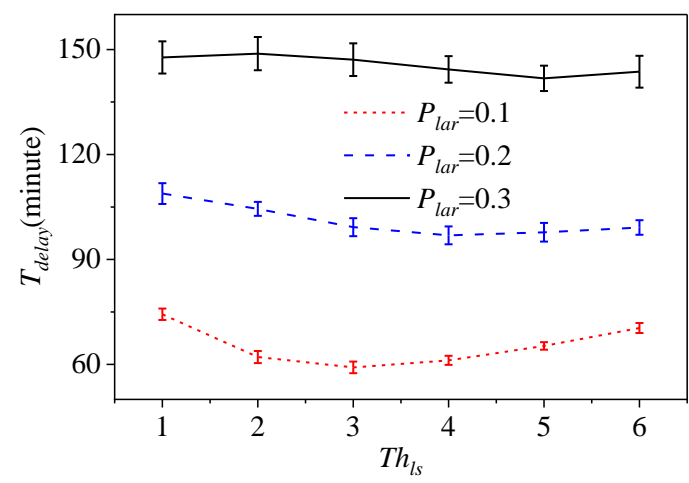

(d) $T_{\text {delay }}$ of all ships in $R_{\text {arr }}=4 h^{-1}$

Fig. 5 Average delay time of ships with different $T h_{l s}$

The tendencies of the average delay time of all ships with different values of $R_{\text {arr }}, P_{\text {lar }}$ and $T h_{l s}$ is present in Fig. 5 (c) and Fig. 5 (d). It can be seen that more ships or more proportion of large ships would result in more delays. In addition, although the curvature radiuses are small, turning points always appear in the curves of average delay time when $R_{\text {arr }}$ or $P_{\text {lar }}$ remains unchanged, which reflects that an optimal $T h_{l s}$ should exist in a designed combination of $R_{a r r}$ and $P_{\text {lar }}$. This means that in order to make all ships passing through the waterway as soon as possible, the traffic flow in the opposite direction should be forbidden once there are $T h_{l s}$ large ships are waiting at the anchorage.

It is noticed that the optimum $T h_{l s}$ raises with the increase of $R_{a r r}$ and $P_{l a r}$. For example, when $P_{l a r}=0.25$ and $R_{a r r}$ increases from 3.0 to 4.0 in Fig. 5(c), the optimum $T h_{l s}$ varies from 2 to 4 , which illustrates that a larger group of large ships could promote the traffic efficiency under the higher arrival rate circumstances. While $R_{a r r}=4 h^{-1}$ and $P_{l a r}$ changes from 0.1 to 0.3 in Fig. 5(d), the optimum $T h_{l s}$ varies in the range of 3-5, which proves that the larger group of large ships will contribute to reduce the average delay time of all ships with increasing proportion of large ships.

In order to investigate the principle of the optimum $T h_{l s}$ for all ships under different parameters, the experimental results in 25 scenarios are listed in Tab. 5. "Difference" means the difference between $T_{\text {delay }}$ in FCFS and in HSOS. The results indicate that there is a close correlation between the optimum $T h_{l s}$ and the two parameters. When ships arrive at a lower frequency, one large ship as a group is enough. The values of delays is also very close to FCFS. When the values of $R_{\text {arr }}$ and $P_{l a r}$ are larger, $T h_{l s}$ should be set as a higher value for obtaining lower $T_{\text {delay }}$ of all ships. 
Tab. 5 Optimum $T h_{l s}$ and $T_{\text {delay }}$ with different $R_{\text {arr }}$ and $P_{\text {lar }}$

\begin{tabular}{|c|c|c|c|c|c|}
\hline $\begin{array}{l}R_{\text {arr }} \\
/ h^{-1}\end{array}$ & $P_{l a r}$ & $\begin{array}{l}\text { optimum } \\
T h_{l s}\end{array}$ & $\begin{array}{l}T_{\text {delay }} \text { in FCFS } \\
\text { Imin }\end{array}$ & $\begin{array}{l}T_{\text {delay }} \\
\text { /min }\end{array}$ in $\mathrm{HSOS}$ & $\begin{array}{l}\text { Difference } \\
\text { /min }\end{array}$ \\
\hline 2 & 0.1 & 1 & 27.66 & 27.61 & 0.05 \\
\hline 2 & 0.15 & 1 & 36.30 & 36.14 & 0.16 \\
\hline 2 & 0.2 & 1 & 42.96 & 42.80 & 0.16 \\
\hline 2 & 0.25 & 1 & 48.91 & 48.59 & 0.32 \\
\hline 2 & 0.3 & 1 & 53.79 & 53.58 & 0.21 \\
\hline 2.5 & 0.1 & 1 & 36.93 & 36.77 & 0.16 \\
\hline 2.5 & 0.15 & 2 & 47.06 & 45.21 & 1.85 \\
\hline 2.5 & 0.2 & 2 & 55.22 & 52.99 & 2.23 \\
\hline 2.5 & 0.25 & 2 & 62.39 & 60.32 & 2.07 \\
\hline 2.5 & 0.3 & 2 & 68.94 & 66.40 & 2.54 \\
\hline 3 & 0.1 & 2 & 47.29 & 42.40 & 4.89 \\
\hline 3 & 0.15 & 2 & 60.14 & 53.91 & 6.23 \\
\hline 3 & 0.2 & 2 & 68.35 & 64.44 & 3.91 \\
\hline 3 & 0.25 & 2 & 77.89 & 73.57 & 4.31 \\
\hline 3 & 0.3 & 3 & 87.25 & 84.09 & 3.16 \\
\hline 3.5 & 0.1 & 2 & 60.11 & 50.80 & 9.31 \\
\hline 3.5 & 0.15 & 3 & 75.04 & 64.70 & 10.34 \\
\hline 3.5 & 0.2 & 3 & 88.13 & 78.26 & 9.87 \\
\hline 3.5 & 0.25 & 3 & 99.72 & 92.24 & 7.48 \\
\hline 3.5 & 0.3 & 3 & 113.16 & 107.05 & 6.11 \\
\hline 4 & 0.1 & 3 & 75.91 & 59.15 & 16.76 \\
\hline 4 & 0.15 & 4 & 95.53 & 78.00 & 17.53 \\
\hline 4 & 0.2 & 4 & 113.46 & 96.89 & 16.57 \\
\hline 4 & 0.25 & 4 & 134.57 & 118.30 & 16.27 \\
\hline 4 & 0.3 & 5 & 157.81 & 141.77 & 16.04 \\
\hline
\end{tabular}

The optimum values of $T h_{l s}$ are imported into HSOS and the performance is analyzed by simulating the traffic efficiency by HSOS and FCFS, respectively. $T_{\text {delay }}$, along with $R_{\text {arr }}, P_{\text {lar }}$ and optimal $T h_{l s}$, are compared comprehensively in the two methods. Until now, HSOS can be performed in an effective way. It can be seen in Fig. 6 (a) that the average delay time nearly grows exponentially with $R_{\text {arr }}$ in FCFS. The average delay time exceeds $1 h$ when $R_{\text {arr }}$ is $2.5 h^{-1}$ and reaches $2.5 h$ when $R_{\text {arr }}$ is $4.0 h^{-1}$. With respect to HSOS, the average delay time is almost the same with FCFS under lower arrival rate. However, there is a declination tendency in HSOS, especially at a higher $R_{\text {arr }}$. In Fig. 6 (b), $T_{\text {delay }}$ nearly grows linearly for FCFS at $R_{\text {arr }}=4 h^{-1}$. The result in HSOS is almost parallel with that in FCFS, and decreased by about $16 \mathrm{~min}$ with the different values of $P_{l a r}$, which is quite remarkable as such a 16-min reduction in delay time could save many transportation costs. It proves that the conflict between the ships in opposite directions has been relieved at a similar extent at a constant $R_{\text {arr }}$ with HSOS. Hence, the expected benefit with HSOS will increase considerably when a larger $R_{a r r}$ is considered. To identify the influence of $R_{\text {arr }}$ and $P_{\text {lar }}$ on $T_{\text {delay }}$ in HSOS, sensitivity analyses [6] are performed. The sensitivity of $R_{\text {arr }}$ is 1.21 while that of $P_{\text {lar }}$ is 0.58 , which also proves that $R_{a r r}$ is more sensitive to $T_{\text {delay }}$ than $P_{l a r}$. In addition, the value of safety distance also has impact on the traffic delays. However, some simulations on different values of safety distance indicate that there is no apparent distinction among the results. Therefore, the results are not present in the paper and we can conclude that safety distance has little correlation with traffic efficiency. 


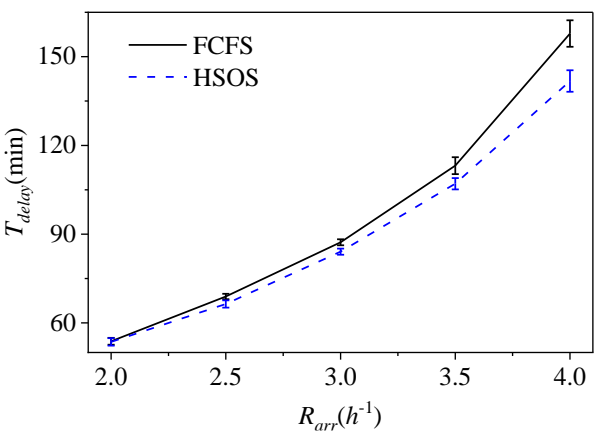

(a) $T_{\text {delay }}$ with $R_{\text {arr }}$ in $P_{\text {lar }}=0.3$

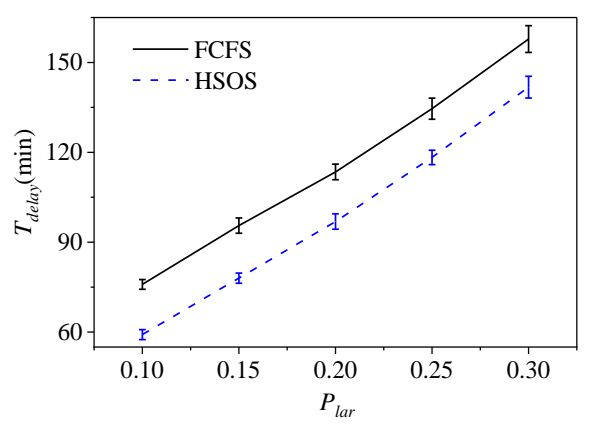

(b) $T_{\text {delay }}$ with $P_{\text {lar }}$ in $R_{\text {arr }}=4 h^{-1}$

Fig. 6 Average delay time of all ships with $R_{a r r}$ and $P_{l a r}$

A comparison of the average delay time of small ships and large ships in FCFS and HSOS is also made in Tab. 6 . The positive difference values stand for that average delay time is decreased in HSOS while the negative values means the increased average delay times. The results show that traffic efficiencies of small ships are promoted in all parameter combinations and the effects are more apparent with the increasing $R_{a r r}$. In the meantime, the delay time of large ships increased in most instances.

Tab. 6 Average delay time of small ships and large ships in FCFS and HSOS

\begin{tabular}{|c|c|c|c|c|c|c|c|}
\hline \multirow{2}{*}{$\begin{array}{l}R_{\text {arr }} \\
\left(h^{-1}\right)\end{array}$} & \multirow{2}{*}{$P_{\text {lar }}$} & \multicolumn{3}{|c|}{$T_{\text {delay }}$ of small ships (min) } & \multicolumn{3}{|c|}{$T_{\text {delay }}$ of large ships ( $\mathrm{min}$ ) } \\
\hline & & FCFS & HSOS & Difference & FCFS & HSOS & Difference \\
\hline 2 & 0.1 & 24.80 & 24.77 & 0.03 & 53.21 & 52.96 & 0.25 \\
\hline 2 & 0.15 & 32.40 & 32.30 & 0.11 & 58.01 & 57.56 & 0.45 \\
\hline 2 & 0.2 & 38.31 & 38.22 & 0.09 & 61.30 & 60.85 & 0.44 \\
\hline 2 & 0.25 & 43.57 & 43.40 & 0.17 & 64.78 & 64.01 & 0.77 \\
\hline 2 & 0.3 & 47.90 & 47.89 & 0.00 & 67.44 & 66.76 & 0.68 \\
\hline 2.5 & 0.1 & 33.84 & 33.69 & 0.14 & 64.54 & 64.27 & 0.28 \\
\hline 2.5 & 0.15 & 42.98 & 32.79 & 10.19 & 69.83 & 114.66 & -44.82 \\
\hline 2.5 & 0.2 & 50.43 & 41.24 & 9.19 & 74.15 & 99.41 & -25.27 \\
\hline 2.5 & 0.25 & 56.83 & 49.01 & 7.82 & 78.96 & 94.06 & -15.10 \\
\hline 2.5 & 0.3 & 62.68 & 55.85 & 6.83 & 83.47 & 90.9 & -7.43 \\
\hline 3 & 0.1 & 44.13 & 31.97 & 12.16 & 75.57 & 135.77 & -60.19 \\
\hline 3 & 0.15 & 56.06 & 43.83 & 12.23 & 83.02 & 110.42 & -27.40 \\
\hline 3 & 0.2 & 63.46 & 55.02 & 8.43 & 87.73 & 101.75 & -14.02 \\
\hline 3 & 0.25 & 71.98 & 64.83 & 7.15 & 95.52 & 99.69 & -4.18 \\
\hline 3 & 0.3 & 80.58 & 70.37 & 10.21 & 102.79 & 116.04 & -13.25 \\
\hline 3.5 & 0.1 & 57.12 & 42.0 & 15.12 & 86.97 & 130.27 & -43.30 \\
\hline 3.5 & 0.15 & 71.19 & 49.6 & 21.60 & 96.73 & 149.75 & -53.02 \\
\hline 3.5 & 0.2 & 83.51 & 64.98 & 18.53 & 106.55 & 131.12 & -24.58 \\
\hline 3.5 & 0.25 & 94.23 & 81.33 & 12.90 & 116.15 & 124.89 & -8.74 \\
\hline 3.5 & 0.3 & 106.79 & 98.46 & 8.33 & 127.98 & 127.03 & 0.95 \\
\hline 4 & 0.1 & 73.11 & 44.94 & 28.17 & 101.38 & 188.15 & -86.77 \\
\hline 4 & 0.15 & 91.99 & 59.0 & 32.99 & 115.55 & 185.51 & -69.96 \\
\hline 4 & 0.2 & 109.63 & 81.8 & 27.83 & 128.75 & 157.15 & -28.4 \\
\hline 4 & 0.25 & 130.55 & 107.93 & 22.61 & 146.63 & 149.4 & -2.77 \\
\hline 4 & 0.3 & 153.83 & 132.57 & 21.26 & 167.10 & 163.18 & 3.92 \\
\hline
\end{tabular}


The tendencies of average delay of small ships and large ships when $R_{\text {arr }}=4 h^{-1}$ are present in Fig. 10. It can be seen that $T_{\text {delay }}$ of small and large ships are almost increasing linearly with $P_{l a r}$ in FCFS. For small ships, the delay time in HSOS are always less than that in FCFS in Fig. 7 (a). The delay of large ships in HSOS are more than that in FCFS and decreased with $P_{l a r}$ apparently in Fig. 7 (b). Although an inflection point appears when $P_{l a r}$ is around 0.25 in HSOS, it is favorable that $T_{\text {delay }}$ of large ships in HSOS is less than that in FCFS when $P_{l a r}$ is 0.3 , which proves that both small ships and large ships obtain benefits when the values of $R_{a r r}$ and $P_{l a r}$ are both high.

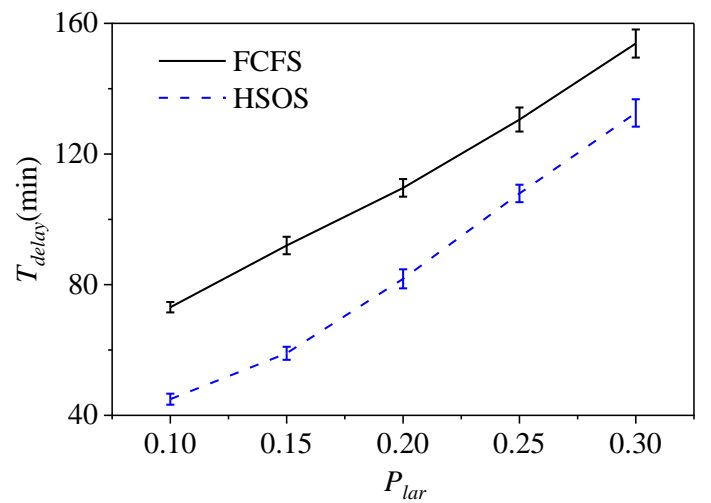

(a) $T_{\text {delay }}$ of small ships in $R_{\text {arr }}=4 h^{-1}$

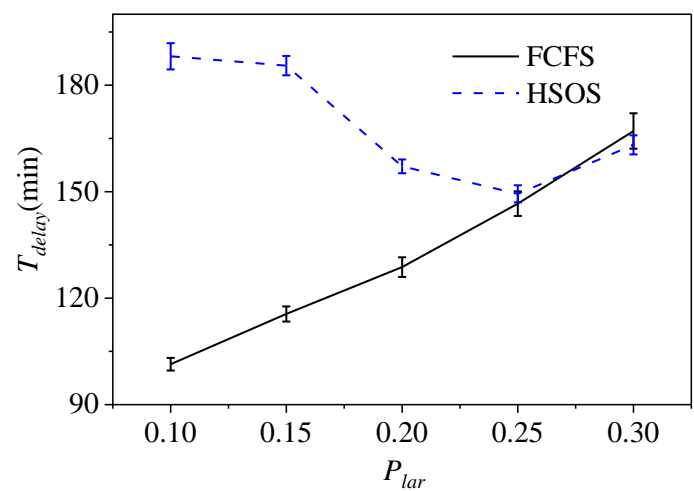

(b) $T_{\text {delay }}$ of large ships in $R_{\text {arr }}=4 h^{-1}$

Fig. 7 Average delay time of small ships and large ships with different $P_{\text {lar }}$ when $R_{\text {arr }}=4 h^{-1}$

\section{Conclusion}

The HSOS method, a self-organizing strategy, is proposed in this paper for ships in restricted two-way waterway. The arriving ships are classified into small ships and large ships in order to define the conflict between them based on ship domain model. The proposed priority scheduling is based on local rules for VTS, corresponding to the detection of the presence (and absence) of large ships waiting at anchorages and rules that assign priority to small ships or large ships. VTS controls the direction of traffic flow on the basis of the number of large ships waiting at an anchorage. The optimum numbers of ships under various circumstances are identified according to the arrival rates and proportion of large ships. Comparing HSOS with FCFS, HSOS can effectively reduce the average delay suffered from the conflicted ships, especially at high arrival rates. Using HSOS, VTS could dynamically adjust to the direction of traffic flow to promote traffic efficiency by adapting to the real-time traffic flow parameters.

It should be noticed that different arrival rules usually occur at two directions in real waterways. In a future work, further discussion can be performed on whether HSOS method is suitable for the unbalanced traffic flow. In addition, cooperation model for multiple legs of waterway can be considered in a wide-range area in order to make the self-organizing scheduling mechanism more practicable and flexible.

\section{ACKNOWLEDGEMENT}

The paper was financially supported by National Key Technologies Research \& Development Program (2017YFE0118000), Science and Technology Program of Zhanjiang (2019A01041, 2019B01012), Project of Enhancing School with Innovation of Guangdong Ocean University $(230419103,230419104)$, program for scientific research start-up funds of Guangdong Ocean University (R20026), Guangxi Natural Science Foundation 
(2018JJB160093), and Qinzhou Science Research and Technology Development Project (20198521),

\section{REFERENCES}

[1] Kim, U. S., Lee, S. W., and Yang, C. H., 2009, "Risk Analysis of VLCS based on Trend of Cargo Growth," Asian Journal of Shipping \& Logistics, 25(1), pp. 19-39.

https://doi.org/10.1016/s2092-5212(09)80010-2

[2] C. Wan, Z. Yang, D. Zhang, et al. "Resilience in transportation systems: a systematic review and future directions." Trans. Rev., vol. 38, no. 4, pp. 479-498, 2018.

https://doi.org/10.1080/01441647.2017.1383532

[3] Meisel, F., and Fagerholt, K., 2019, "Scheduling two-way ship traffic for the Kiel Canal: Model, extensions and a matheuristic," Computers \& Operations Research, 106, pp. 119-132. https://doi.org/10.1016/j.cor.2019.02.015

[4] Heyligen, F., 1990, "Classical and nonclassical representations in physics: physics 1," Cybernetics and Systems: An International Journal, 21(4), pp. 423-444. https://doi.org/10.1080/01969729008902251

[5] Zhang, X., Lin, J., Guo, Z., and Liu, T., 2016, "Vessel transportation scheduling optimization based on channel-berth coordination," Ocean Eng, 112, pp. 145-152.

https://doi.org/10.1016/j.oceaneng.2015.12.011

[6] Zhang, X. Y., Chen, X., Ji, M. J., and Yao, S., 2017, "Vessel Scheduling Model of a One-Way Port Channel," J Waterw Port Coast, 143(5), p. 04017009.

https://doi.org/10.1061/(Asce)Ww.1943-5460.0000388

[7] Wang, H., Liu, J., Liu, K., Zhang, J., and Wang, Z., 2018, "Sensitivity analysis of traffic efficiency in restricted channel influenced by the variance of ship speed," Proceedings of the Institution of Mechanical Engineers, Part M: Journal of Engineering for the Maritime Environment, 232(2), pp. 212-224.

https://doi.org/10.1177/1475090217720549

[8] Liang, S., Yang, X., Bi, F., and Ye, C., 2019, "Vessel traffic scheduling method for the controlled waterways in the upper Yangtze River," Ocean Eng, 172, pp. 96-104.

https://doi.org/10.1016/j.oceaneng.2018.11.025

[9] Zhang, J. F., Santos, T. A., Guedes Soares, C., and Yan, X. P., 2017, "Sequential ship traffic scheduling model for restricted two-way waterway transportation," Proceedings of the Institution of Mechanical Engineers Part M: Journal of Engineering for the Maritime Environment, 231 (1), pp. 86-97.

https://doi.org/10.1177/1475090215621580

[10] Coomber, F. G., D'Inca, M., Rosso, M., Tepsich, P., di Sciara, G. N., and Moulins, A., 2016, "Description of the vessel traffic within the north Pelagos Sanctuary: Inputs for Marine Spatial Planning and management implications within an existing international Marine Protected Area," Mar Policy, 69, pp. 102-113. https://doi.org/10.1016/j.marpol.2016.04.013

[11] Xin, X., Liu, K., Yang, X., Yuan, Z., and Zhang, J., 2019, "A simulation model for ship navigation in the "Xiazhimen" waterway based on statistical analysis of AIS data," Ocean Eng, 180, pp. 279-289. https://doi.org/10.1016/j.oceaneng.2019.03.052

[12] Kauffman, S. A., 1992, "Origins of order in evolution: self-organization and selection," Understanding Origins, Springer, pp. 153-181. https://doi.org/10.1007/978-94-015-8054-0_8

[13] Westrenen, F. V., and Praetorius, G., 2014, "Maritime traffic management: a need for central coordination?," Cogn Technol Work, 16(1), pp. 59-70. https://doi.org/10.1007/s10111-012-0244-5

[14] Berezin, A. A., and Gridin, V. V., 2017, "Isotopic Self-Organization as an Informational Factor in Biological Systems," Nonlin Dynam Psychol, 21(4), pp. 485-504.

http://europepmc.org/abstract/MED/28923158

[15] Ferreira, M., Fernandes, R., Conceição, H., Viriyasitavat, W., and Tonguz, O. K., 2010, "Self-organized traffic control," Proceedings of the seventh ACM international workshop on VehiculAr InterNETworking, ACM, Chicago, Illinois, USA, pp. 85-90. https://doi.org/10.1145/1860058.1860077

[16] Gershenson, C., and Rosenblueth, D. A., 2012, "Self-organizing traffic lights at multiple-street intersections," Complexity, 17(4), pp. 23-39. Gershenson, C., \& Rosenblueth, D. A. (2011). Selforganizing traffic lights at multiple-street intersections. Complexity, 17(4), 23-39. 
https://doi.org/10.1002/cplx.20392

[17] Tonguz, O. K., and Viriyasitavat, W., 2016, "A self-organizing network approach to priority management at intersections," IEEE Communications Magazine, 54(6), pp. 119-127.

https://doi.org/10.1109/MCOM.2016.7498098

[18] Wolfram, S., 1984, "Cellular automata as models of complexity," Nature, 311(5985), pp. 419-424. https://doi.org/10.1038/311419a0

[19] Qu, X. B., and Meng, Q., 2012, "Development and applications of a simulation model for vessels in the Singapore Straits," Expert Syst Appl, 39(9), pp. 8430-8438. https://doi.org/10.1016/j.eswa.2012.01.176

[20] Statheros, T., Howells, G., and Maier, M. D., 2008, "Autonomous Ship Collision Avoidance Navigation Concepts, Technologies and Techniques," J Navigation, 61(1), pp. 129-142.

https://doi.org/10.1017/s037346330700447x

[21] B. Wu, T. Leung, X. Yan, and C. G. Soares, "Fuzzy logic based approach for ship-bridge collision alert system," Ocean Eng., vol. 187, p. 106152, 2019. https://doi.org/10.1016/j.oceaneng.2019.106152

[22] B. Wu, T. Cheng, T. Yip, et al. "Fuzzy logic based dynamic decision-making system for intelligent navigation strategy within inland traffic separation schemes." Ocean Eng., vol. 197, no. February 106909. 2020. https://doi.org/10.1016/j.oceaneng.2019.106909

[23] J. Zhang, Â. Teixeira, C. Guedes Soares, X. Yan., and K. Liu, "Maritime Transportation Risk Assessment of Tianjin Port with Bayesian Belief Networks," Risk Anal., vol. 36, no. 6, pp. 1171-1187, 2016. https://doi.org/10.1111/risa.12519

[24] Vaněk, O., Jakob, M., Hrstka, O., and Pěchouček, M., 2013, "Agent-based model of maritime traffic in piracy-affected waters," Transportation Research Part C: Emerging Technologies, 36(11), pp. 157-176. https://doi.org/10.1016/j.trc.2013.08.009

[25] Wang, H., Liu, J., Zhang, J., Liu, K., Yang, X., and Yu, Q., 2018, "Self-organizing cooperation model for ships navigating in restricted one-way waterway," Brodogradnja, 69(3), pp. 1-23. https://doi.org/10.21278/brod69301

[26] Szlapczynski, R., and Szlapczynska, J., 2017, "Review of ship safety domains: Models and applications," Ocean Eng, 145, pp. 277-289. https://doi.org/10.1016/j.oceaneng.2017.09.020

[27] Liu, J., Feng, Z., Li, Z., Wang, M., and Wen, L. R., 2016, "Dynamic Ship Domain Models for Capacity Analysis of Restricted Water Channels," J Navigation, 69(3), pp. 481-503. https://doi.org/10.1017/s0373463315000764

[28] Mou, J. M., Tak, C. v. d., and Ligteringen, H., 2010, "Study on collision avoidance in busy waterways by using AIS data," Ocean Eng, 37(5), pp. 483-490. https://doi.org/10.1016/j.oceaneng.2010.01.012

[29] Zubillaga, D., Cruz, G., Aguilar, L., Zapotécatl, J., Fernández, N., Aguilar, J., Rosenblueth, D., and Gershenson, C., 2014, "Measuring the complexity of self-organizing traffic lights," Entropy, 16(5), pp. 2384-2407. https://doi.org/10.3390/e16052384

[30] Wang, W., Peng, Y., Tian, Q., and Song, X., 2017, "Key influencing factors on improving the waterway through capacity of coastal ports," Ocean Eng, 137, pp. 382-393.

https://doi.org/10.1016/j.oceaneng.2017.04.013

Submitted: $\quad$ 07.01.2020.

Accepted: $\quad$ 17.04.2020.
Hongbo Wang,whonbo@hotmail.com

Maritime College, Guangdong Ocean University, Zhanjiang, China

Wuliu Tian*, Corresponding Author, tianwuliu@ foxmail.com

Maritime college, Beibu Gulf University, Qinzhou, China

Jinfen Zhang, jinfen.zhang@whut.edu.cn

National Engineering Research Center for Water Transport Safety, Wuhan University of Technology, Wuhan, China

Yongjiang Li, lyjriver@126.com

School of Mathematics and Computer, Guangdong Ocean University, Zhanjiang, China 